\title{
Phase diagram of Yukawa systems near the one-component-plasma limit revisited
}

\author{
S. Hamaguchi \\ IBM, Thomas J. Watson Research Center, P.O. Box 218, Yorktown Heights, New York 10598 \\ R. T. Farouki \\ Department of Mechanical Engineering and Applied Mechanics, University of Michigan, Ann Arbor, \\ Michigan 48109 \\ D. H. E. Dubin \\ Department of Physics, University of California at San Diego, La Jolla, California 92093
}

(Received 12 February 1996; accepted 25 July 1996)

Transition inverse temperatures (or $\Gamma$ values) at the fluid-solid phase boundary of Yukawa systems near the one-component-plasma (OCP) limit have been evaluated by molecular dynamics simulations. These values are systematically smaller than those obtained in an earlier study by Farouki and Hamaguchi [J. Chem. Phys. 101, 9885 (1994)]. The discrepancy is attributed to the fact that, in the earlier study, the harmonic entropy constants were approximated by that of the OCP, whereas the new results are based on more accurate harmonic entropy constants obtained from lattice-dynamics calculations. The new molecular dynamics simulations also confirm that the becfcc phase transition curve is in good agreement with that of the quasiharmonic theory in the regime $\kappa \leqslant 1.4$, where $\kappa$ is the ratio of the Wigner-Seitz radius to the Debye length. Examples of Yukawa systems include dusty plasmas and colloidal suspensions. (C) 1996 American Institute of Physics. [S0021-9606(96)51541-2]

\section{INTRODUCTION}

Plasmas containing small solid particles, i.e., "dusty" plasmas, have attracted much attention recently. In the semiconductor industry, such particles are known to cause damage to substrates during plasma processing. In space plasmas, the existence of such particles affects various optical observations.

Small particles in a plasma are typically negatively charged, due to the high mobility of electrons, and they interact with each other through a Yukawa-type (i.e., screened Coulomb) pair potential [see Eq. (1) below]. Laboratory experiments have recently demonstrated that, when the interparticle potential energy exceeds the kinetic energy, particulates in plasmas may form crystalline structures. ${ }^{1-7}$ The Yukawa system is also known to model colloidal particles suspended in electrolyte solutions. Crystalline structures of colloids are also commonly observed in experiments. ${ }^{8}$

In a recent study by Farouki and Hamaguchi, ${ }^{9}$ the fluidsolid (i.e., freezing-melting) phase transition curve was obtained for Yukawa systems near the one-component-plasma (OCP) limit, based on molecular dynamics (MD) simulations. In their derivation of the phase-transition curve, however, those authors used an approximate value for the harmonic entropy constants $\Sigma(\kappa)$ [defined in Eq. (10) in Sec. II below] of Yukawa systems. The goal of this paper is to reevaluate the phase-transition curve using more accurate values of the harmonic entropy constants obtained from lattice dynamics calculations, and thus to present more accurate transition temperatures (or $\Gamma$ values).

As in Ref. 9, we consider a system of identical particles of mass $m$, charge $Q=-Z e(Z \gg 1)$ immersed in a neutraliz- ing background plasma. The interparticle potential is of Yukawa type, given by

$$
\phi(r)=\frac{Q^{2}}{4 \pi \epsilon_{0} r} \exp \left(-k_{D} r\right)
$$

where $r$ denotes the radial distance between two particles. The Debye length $\lambda_{D}=k_{D}^{-1}$ of the background plasma is defined by

$$
\lambda_{D}=\left(\frac{q_{i}^{2} \bar{n}_{i}}{\epsilon_{0} k T_{i}}+\frac{e^{2} \bar{n}_{e}}{\epsilon_{0} k T_{e}}\right)^{-1 / 2},
$$

with $q_{i}, \overline{n_{i}}$, and $T_{i}$ being the charge, mean density, and temperature of plasma ions, and $-e, \bar{n}_{e}$, and $T_{e}$ the corresponding quantities for plasma electrons.

Using the Wigner-Seitz radius $a=(3 / 4 \pi n)^{1 / 3}$ as the unit of length, where $n$ is the particle number density, we may describe the thermodynamics of the Yukawa system in terms of two dimensionless ratios:

$$
\kappa=\frac{a}{\lambda_{D}}, \quad \Gamma=\frac{Q^{2}}{4 \pi \epsilon_{0} a k T} .
$$

Note that parameter $\Gamma$ is roughly the ratio of the (unscreened) Coulomb potential energy to the kinetic energy per particle.

In this paper, we focus on the regime of weak Debye screening $(\kappa \lesssim 1)$ as in Ref. 9, which is relevant to experimental observations of strongly coupled particulate systems in plasmas. In the limit $\kappa \rightarrow 0$, the Yukawa system becomes the OCP (a system of mobile charges immersed in a strictly uniform neutralizing background), which has been extensively 
studied $^{10-16}$ as a model for the interior of white dwarf stars. In this sense, the Yukawa system may be considered a classical generalization of the OCP.

\section{FREE ENERGY CALCULATIONS}

We employ the MD technique and free energy calculation method described in Ref. 9. We briefly summarize these methods in this section.

For MD simulations in the week-screening regime, where the range of the interparticle force becomes comparable to or greater than the side $L$ of the cubical simulation volume, one must use the effective pair potential

$$
\boldsymbol{\Phi}(\mathbf{r})=\phi(|\mathbf{r}|)+\sum_{\mathbf{n} \neq \mathbf{0}} \phi(|\mathbf{r}+\mathbf{n} L|),
$$

which reflects periodic boundary conditions imposed on the simulation box, to emulate correct particle-particle interactions. In the above equation, $\boldsymbol{\Phi}(\mathbf{r})$ represents the interaction energy of particle $i$ with particle $j$ (at separation $\mathbf{r}=\mathbf{r}_{j}-\mathbf{r}_{i}$ ) and with all periodic images of the latter. The infinite sum of $\phi$ over integer vectors $\mathbf{n}=(l, m, n)$ represents the periodic images. Numerically this periodic image potential is approximated by a tensor-product spline function interpolating an array of $40 \times 40 \times 40$ discrete values, summed to high accuracy. The approximation can be efficiently evaluated in the simulations and has a fractional deviation from the exact value of no more than $\sim 10^{-7}$. Full details of the approximation scheme may be found in Ref. 17.

The total potential energy (or "excess energy") $U$ of the model system with periodic boundary conditions is then given by

$$
\begin{aligned}
\frac{U}{N k T}= & \Gamma\left[\frac{1}{N} \sum_{j=1}^{N-1} \sum_{k=j+1}^{N} \hat{\Phi}\left(\boldsymbol{\xi}_{k}-\boldsymbol{\xi}_{j}\right)-\frac{3}{2 \kappa^{2}}-\frac{\kappa}{2}\right. \\
& \left.+\frac{1}{2} \sum_{\mathbf{n} \neq \mathbf{0}} \frac{\exp (-\kappa|\mathbf{n}| \Lambda)}{|\mathbf{n}| \Lambda}\right],
\end{aligned}
$$

where $N$ is the number of particles, $\Lambda=L / a=(4 \pi N / 3)^{1 / 3}$ is the size of the cubical simulation volume in units of the Wigner-Seitz radius, $\boldsymbol{\xi}_{i}=\mathbf{r}_{i} / a$ is the dimensionless location of particle $i$, and $\hat{\boldsymbol{\Phi}}=4 \pi \epsilon_{0} a \boldsymbol{\Phi} / Q^{2}$.

The energy associated with the background plasma in Eq. (4) is not just the potential energy, but the free energyincluding the contribution of the entropy associated with thermal motions of the plasma ions and electrons. Thus, the partial derivative of $U$ with respect to $\mathbf{r}_{i}$ gives the true force on the $i$ th particle. ${ }^{18}$ In Eq. (4), the second term inside the square bracket represents the free energy (excluding the uniform ideal-gas free energy) of the background plasma that, on average, neutralizes the charge of the particulates. The third term represents the free energy of each Debye sheath [see Eq. (17) of Ref. 19], and the fourth term represents the energy of interaction of each particulate with its own images under periodic boundary conditions.

As $\kappa \rightarrow 0$, the first, second, and last terms on the righthand side of Eq. (4) diverge in such a manner that the limiting value of $U$ equals the well-known expression $U_{\mathrm{OCP}}$ for the OCP potential energy given by, for example, Eqs. (6) and (8) of Ref. 9. As the screening length increases (i.e., as $\kappa \rightarrow 0$ ), each particulate has an increasingly strong interaction with both the charge-neutralizing background plasma and the other charged particulates. Thus, the magnitude of the background-plasma free energy - the second term in Eq. (4) - increases to cancel the increase of direct electrostatic interactions among charged particles, i.e., the first and last terms in Eq. (4).

To see the correspondence between the Yukawa system and OCP system more clearly, one may write

$$
U=\sum_{j=1}^{N-1} \sum_{k=j+1}^{N} \psi\left(\mathbf{r}_{k}-\mathbf{r}_{j}\right)+U_{0}
$$

where

$$
\frac{\psi(\mathbf{r})}{N k T}=\frac{\Gamma}{N}\left[\hat{\Phi}(\boldsymbol{\xi})-\frac{3}{\kappa^{2} N}\right],
$$

and

$$
\frac{U_{0}}{N k T}=\frac{\Gamma}{2}\left[\sum_{\mathbf{n} \neq \mathbf{0}} \frac{\exp (-\kappa|\mathbf{n}| \Lambda)}{|\mathbf{n}| \Lambda}-\frac{3}{\kappa^{2} N}-\kappa\right] .
$$

Then, in the OCP limit $\kappa \rightarrow 0$, one can show that the pair potential $\psi$ and energy constant $U_{0}$ converge to those of the OCP, i.e., $\psi \longrightarrow \psi^{\mathrm{OCP}}$ and $U_{0} \rightarrow U_{0}^{\mathrm{OCP}}$, where

$$
\psi^{\mathrm{OCP}}(\mathbf{r})=\frac{Q^{2}}{4 \pi \epsilon_{0}} \int_{V_{\infty}}\left[\sum_{\mathbf{n}} \delta(\boldsymbol{\rho}-\mathbf{n} L)-\frac{1}{L^{3}}\right] \frac{1}{|\boldsymbol{\rho}-\mathbf{r}|} d \boldsymbol{\rho}
$$

and

$$
\begin{aligned}
U_{0}^{\mathrm{OCP}} & =\frac{1}{2} N \lim _{|\mathbf{r}| \rightarrow 0}\left(\psi^{\mathrm{OCP}}(\mathbf{r})-\frac{Q^{2}}{4 \pi \epsilon_{0}|\mathbf{r}|}\right) \\
& \approx-1.4186487 \frac{Q^{2} N}{4 \pi \epsilon_{0} L} .
\end{aligned}
$$

For further details, the reader is referred to Ref. 18.

We denote the internal energy and Helmholtz free energy per particle in units of $k T$ by

$$
u=\frac{U}{N k T}, \quad f=\frac{F}{N k T} .
$$

The thermal component of the potential energy is defined by

$$
u_{\mathrm{th}}(\kappa, \Gamma)=u(\kappa, \Gamma)-u_{\infty}(\kappa),
$$

where $u_{\infty}(\kappa)$ represents the Madelung energy (for an appropriate lattice) per particle in units of $k T$. We also define

$$
E(\kappa)=\lim _{\Gamma \rightarrow \infty} \frac{u(\kappa, \Gamma)}{\Gamma},
$$

i.e., the Madelung energy per particle in units of $Q^{2} / 4 \pi \epsilon_{0} a$, so that $u_{\infty}(\kappa)=E(\kappa) \Gamma$. In the limit of zero temperature (i.e., $\Gamma \rightarrow \infty)$, we evidently have $u(\kappa, \infty)=u_{\infty}(\kappa)$. At zero temperature, the bcc Madelung energy is smaller than the fcc Madelung energy ${ }^{9}\left[E_{\mathrm{bcc}}(\kappa)<E_{\mathrm{fcc}}(\kappa)\right.$ for $\left.\kappa<1.066\right]$. The bcc-fcc phase transition can therefore occur only for $\kappa>1.066$. 
TABLE I. Harmonic entropy constants for bcc and fcc Yukawa lattices. The values at $\kappa=0$ are quoted from Ref. 21

\begin{tabular}{ccc}
\hline \hline$\kappa$ & $\Sigma_{\mathrm{bcc}}$ & $\Sigma_{\mathrm{fcc}}$ \\
\hline 0.0 & -2.4939 & -2.4537 \\
0.2 & -2.5172 & -2.4767 \\
0.4 & -2.5808 & -2.5393 \\
0.6 & -2.6829 & -2.6394 \\
0.8 & -2.8185 & -2.7722 \\
1.0 & -2.9843 & -2.9347 \\
1.2 & -3.1773 & -3.1236 \\
1.4 & -3.3950 & -3.3366 \\
\hline \hline
\end{tabular}

Since $\partial f / \partial \Gamma=u(\kappa, \Gamma) / \Gamma$, the dimensionless Helmholtz free energy for the fluid phase may be defined by

$$
f_{\text {fluid }}(\kappa, \Gamma)=\int_{0}^{\Gamma} u\left(\kappa, \Gamma^{\prime}\right) \frac{d \Gamma^{\prime}}{\Gamma^{\prime}}+f_{\text {ideal }}(\Gamma) .
$$

Here the last term represents the ideal-gas contribution to the total free energy, i.e.,

$$
\begin{aligned}
f_{\text {ideal }}(\Gamma) & =\ln \left[\left(\frac{2 \pi \hbar^{2}}{m k T}\right)^{3 / 2} n\right]-1 \\
& =3 \ln \Gamma+\frac{3}{2} \ln (k T)_{\mathrm{Ry}}-1+\ln \frac{3 \sqrt{\pi}}{4},
\end{aligned}
$$

where $(k T)_{\text {Ry }}$ denotes $k T$ measured in Rydberg units, $\frac{1}{2}\left(Q^{2} / 4 \pi \epsilon_{0} \hbar\right)^{2} m$, for the particle. Although $f_{\text {ideal }}$ depends on $(k T)_{\mathrm{Ry}}$ as well as $\Gamma$, we do not explicitly express the dependence on the former for the sake of simplicity.

For the solid phase, we use

TABLE II. Equilibrium potential energy per particle, $u / \Gamma$, in the fluid phase, $(N=500)$. The numbers after \pm indicate fluctuation levels. Note that $u / \Gamma$ $\rightarrow-\kappa / 2$ as $\Gamma \rightarrow 0$

\begin{tabular}{rlc}
\hline \hline$\Gamma$ & $\kappa=1.2$ & $\kappa=1.4$ \\
\hline 0.00 & -0.600000 & -0.700000 \\
0.10 & $-0.648425 \pm 0.025551$ & $-0.740415 \pm 0.023959$ \\
0.20 & $-0.680595 \pm 0.024165$ & $-0.770037 \pm 0.021136$ \\
0.40 & $-0.724539 \pm 0.019526$ & $-0.806296 \pm 0.016947$ \\
0.60 & $-0.757346 \pm 0.015638$ & $-0.831754 \pm 0.015606$ \\
0.80 & $-0.782004 \pm 0.013549$ & $-0.852470 \pm 0.012292$ \\
1 & $-0.797097 \pm 0.010904$ & $-0.869746 \pm 0.011675$ \\
2 & $-0.855439 \pm 0.009179$ & $-0.920097 \pm 0.008272$ \\
5 & $-0.921138 \pm 0.004200$ & $-0.978642 \pm 0.004510$ \\
10 & $-0.958561 \pm 0.002799$ & $-1.012022 \pm 0.002587$ \\
20 & $-0.985481 \pm 0.001533$ & $-1.037095 \pm 0.001467$ \\
40 & $-1.004536 \pm 0.000893$ & $-1.055146 \pm 0.000911$ \\
60 & $-1.012768 \pm 0.000683$ & $-1.062759 \pm 0.000616$ \\
80 & $-1.017387 \pm 0.000559$ & $-1.067144 \pm 0.000495$ \\
100 & $-1.020683 \pm 0.000433$ & $-1.070322 \pm 0.000426$ \\
120 & $-1.022726 \pm 0.000427$ & $-1.072513 \pm 0.000409$ \\
140 & $-1.024535 \pm 0.000353$ & $-1.074088 \pm 0.000329$ \\
160 & $-1.025919 \pm 0.000310$ & $-1.075469 \pm 0.000253$ \\
180 & $-1.027188 \pm 0.000249$ & $-1.076467 \pm 0.000301$ \\
200 & $-1.028005 \pm 0.000250$ & $-1.077485 \pm 0.000250$ \\
240 & $\cdots$ & $-1.078874 \pm 0.000204$ \\
\hline \hline
\end{tabular}

TABLE III. Equilibrium potential energy per particle, $u / \Gamma$, for bcc solids $(N=686)$. The numbers after \pm indicate fluctuation levels. The energy value with an asterisk $\left(^{*}\right)$ was not used for fitting since the value clearly deviates from either fitting curve, indicating the system is in a mixed fluid-solid state.

\begin{tabular}{rcc}
\hline \hline$\Gamma$ & $\kappa=1.2$ & $\kappa=1.4$ \\
\hline 240 & $-1.032521^{*} \pm 0.000184$ & $\ldots$ \\
300 & $-1.034044 \pm 0.000117$ & $-1.083042 \pm 0.000134$ \\
400 & $-1.035414 \pm 0.000103$ & $-1.084449 \pm 0.000099$ \\
500 & $-1.036214 \pm 0.000072$ & $-1.085260 \pm 0.000088$ \\
600 & $-1.036742 \pm 0.000064$ & $-1.085788 \pm 0.000054$ \\
800 & $-1.037388 \pm 0.000045$ & $-1.086444 \pm 0.000042$ \\
1000 & $-1.037774 \pm 0.000044$ & $-1.086828 \pm 0.000038$ \\
$\infty$ & -1.039292 & -1.088350 \\
\hline \hline
\end{tabular}

$$
f_{\text {solid }}(\kappa, \Gamma)=\int_{\infty}^{\Gamma}\left[u_{\text {th }}\left(\kappa, \Gamma^{\prime}\right)-\frac{3}{2}\right] \frac{d \Gamma^{\prime}}{\Gamma^{\prime}}+f_{\text {harm }}(\kappa, \Gamma),
$$

where $u_{\mathrm{th}}-3 / 2$ is the anharmonic component of the potential energy in units of $k T$. The free energy for the harmonic lattice vibration is given by

$$
\begin{aligned}
f_{\text {harm }}(\kappa, \Gamma)= & E(\kappa) \Gamma+\Sigma(\kappa)+\frac{9}{2} \ln \Gamma+\frac{3}{2} \ln (k T)_{\text {Ry }} \\
& +\frac{3}{2} \ln \frac{3}{2},
\end{aligned}
$$

where $\Sigma(\kappa)$ denotes the harmonic entropy constant, i.e.,

$$
\Sigma(\kappa)=\lim _{N \rightarrow \infty} \frac{1}{N} \sum_{k=1}^{3 N-3} \ln \frac{\omega_{k}}{\omega_{p}} .
$$

Here $\omega_{p}=\sqrt{Q^{2} n / \epsilon_{0} m}$ is the plasma frequency of the particles and the sum is taken over the $3 N-3$ normal-mode frequencies $\omega_{k}$ for the oscillation of a lattice of $N$ particles. The eigenfrequencies $\omega_{k}$ of an $N$-particle Yukawa lattice may be computed using standard techniques, ${ }^{20}$ and the quantity $\Sigma(\kappa)$ can then be estimated for various $\kappa$ values by letting $N \rightarrow \infty$. Table I gives the values of $\Sigma(\kappa)$ for bcc and fcc Yukawa lattices. The OCP values (i.e., $\kappa=0$ ) in Table I are taken from Table I of Ref. 21.

\section{PHASE TRANSITION}

MD simulations are used to evaluate the potential energy $u$ for given values of the thermodynamic variables $\kappa$ and $\Gamma$. Details of the simulation method may be found in Refs. 9, 16 , and 17. The number of particles $N$ used for the simulations reported here and in Ref. 9 are $N=686$ for a bcc and $N=500$ for a fcc lattice. These lattices are used as initial conditions, and the system is allowed to equilibrate to the desired $\Gamma$ for 100 time units before averaging its properties over $100<\tau<300$. Here the time unit is defined to be $\sqrt{3} \omega_{p}^{-1}$, so that $\tau=\omega_{p} t / \sqrt{3}$. Cases that melted to a fluid state did so well before $\tau=100$.

For the fluid phase, we fit measured potential energies to the expression

$$
u(\kappa, \Gamma)=a(\kappa) \Gamma+b(\kappa) \Gamma^{s}+c(\kappa)+d(\kappa) \Gamma^{-s},
$$

with $s=1 / 3$. This functional form has been applied to internal energy fitting of various OCP simulations. ${ }^{14}$ Writing 
TABLE IV. Equilibrium potential energy per particle, $u / \Gamma$, for fcc solids $(N=500)$. The numbers after \pm indicate fluctuation levels. The energy value with an asterisk $(*)$ was not used for fitting since the value clearly deviates from either fitting curve, indicating the system is in a mixed fluid-solid state.

\begin{tabular}{rlc}
\hline \hline$\Gamma$ & $\kappa=1.2$ & $\kappa=1.4$ \\
\hline 240 & $-1.032173^{*} \pm 0.000228$ & $\ldots$ \\
300 & $-1.034003 \pm 0.000150$ & $-1.082998 \pm 0.000165$ \\
400 & $-1.035392 \pm 0.000110$ & $-1.084437 \pm 0.000114$ \\
500 & $-1.036209 \pm 0.000085$ & $-1.085274 \pm 0.000091$ \\
600 & $-1.036735 \pm 0.000071$ & $-1.085804 \pm 0.000082$ \\
800 & $-1.037390 \pm 0.000058$ & $-1.086460 \pm 0.000057$ \\
1000 & $-1.037778 \pm 0.000046$ & $-1.086849 \pm 0.000049$ \\
$\infty$ & -1.039302 & -1.088374 \\
\hline \hline
\end{tabular}

$a(\kappa)=E_{\mathrm{bcc}}(\kappa)+\delta a(\kappa)$ as in Ref. 9, we first apply a leastsquares fit to $E_{\mathrm{bcc}}(\kappa)$ data given in Table III of Ref. 9 and obtain

$$
\begin{aligned}
E_{\mathrm{bcc}}(\kappa)= & -0.895929-0.103731 \kappa^{2}+0.003084 \kappa^{4} \\
& -0.000131 \kappa^{6} \text { for } \kappa \leqslant 1 .
\end{aligned}
$$

To fit the fluid data given in Table II of Ref. 9 to expression (11), we take the dependence of the coefficients $\delta a, b, c, d$ on $\kappa$ to be no more than quartic with even powers only, i.e., we write

$$
\delta a(\kappa)=\delta a_{0}+\delta a_{2} \kappa^{2}+\delta a_{4} \kappa^{4},
$$

and similarly for $b(\kappa), c(\kappa)$, and $d(\kappa)$. Twelve term fits of the fluid data over the dual independent variables $\kappa$ and $\Gamma$ yield

$\delta a(\kappa)=-0.003366+0.000660 \kappa^{2}-0.000089 \kappa^{4}$,

$b(\kappa)=0.565004-0.026134 \kappa^{2}-0.002689 \kappa^{4}$,

$c(\kappa)=-0.206893-0.086384 \kappa^{2}+0.018278 \kappa^{4}$,

$d(\kappa)=-0.031402+0.042429 \kappa^{2}-0.008037 \kappa^{4}$,

for $\kappa \leqslant 1$. Here we have employed higher-order least-squares polynomial fits (but with even powers only) than those used $^{22}$ in Ref. 9.

To avoid divergence on substituting expression (11), we write Eq. (6) as

$$
f_{\text {fluid }}(\kappa, \Gamma)=\int_{1}^{\Gamma} u\left(\kappa, \Gamma^{\prime}\right) \frac{d \Gamma^{\prime}}{\Gamma^{\prime}}+f_{1}(\kappa)+f_{\text {ideal }}(\Gamma),
$$

with

$$
f_{1}(\kappa)=\int_{0}^{1} u\left(\kappa, \Gamma^{\prime}\right) \frac{d \Gamma^{\prime}}{\Gamma^{\prime}} .
$$

TABLE V. Fluid fitting parameters $a, b, c$, and $d$ defined by Eq. (11) for $\kappa=1.2$ and 1.4. For $\kappa \leqslant 1.0$, these parameters are given as functions of $\kappa$ by Eqs. (13)-(16). Note that $\delta a(\kappa)$ in Eq. (13) is defined as $\delta a(\kappa)=a(\kappa)-E_{\mathrm{bcc}}(\kappa)$.

\begin{tabular}{ccccc}
\hline \hline$\kappa$ & $a$ & $b$ & $c$ & $d$ \\
\hline 1.2 & -1.041816 & 0.522733 & -0.305649 & 0.026740 \\
1.4 & -1.090801 & 0.514325 & -0.344195 & 0.049258 \\
\hline \hline
\end{tabular}

TABLE VI. Solid fitting parameters $A_{1}$ and $A_{2}$ for bcc and fec Yukawa lattices defined by Eq. (18). For $\kappa \leqslant 1.0, A_{1}(\kappa)$ and $A_{2}(\kappa)$ for bcc Yukawa lattices are given by Eqs. (19) and (20).

\begin{tabular}{ccccc}
\hline \hline$\kappa$ & $A_{1}^{\text {bcc }}$ & $A_{2}^{\text {bcc }}$ & $A_{1}^{\text {fcc }}$ & $A_{2}^{\text {fcc }}$ \\
\hline 1.2 & 15.42 & 2042.56 & 21.13 & 1712.24 \\
1.4 & 16.12 & 3398.78 & 17.87 & 4735.20 \\
\hline \hline
\end{tabular}

[Note that $f_{1}(\kappa)$ is denoted as $f(\kappa, 1)$ in Ref. 9; we employ a new notation here since $f_{\text {fluid }}(\kappa, \Gamma)$ in this paper includes $f_{\text {ideal }}(\Gamma)$ - the ideal gas contribution-whereas the fluidphase free energy $f(\kappa, \Gamma)$ in Ref. 9 is defined to exclude the ideal gas contribution.] The integral $f_{1}(\kappa)$ is evaluated through a direct Simpson-rule quadrature of the $u / \Gamma$ values obtained from MD simulations.

For the solid phase, the following form for the thermal potential energy is assumed:

$$
u_{\mathrm{th}}(\kappa, \Gamma)=\frac{3}{2}+\frac{A_{1}(\kappa)}{\Gamma}+\frac{A_{2}(\kappa)}{\Gamma^{2}},
$$

where $3 / 2$ is the harmonic component, and the power series in $\Gamma^{-1}$ represents the anharmonic terms. From a least-squares fit of the bcc solid phase data given in Table II of Ref. 9 over the dual independent variables $\kappa$ and $\Gamma$, we obtain

$$
\begin{aligned}
& A_{1}(\kappa)=9.13+4.37 \kappa^{2}, \\
& A_{2}(\kappa)=1526+479 \kappa^{2},
\end{aligned}
$$

for bcc lattices with $\kappa \leqslant 1$.

For $\kappa>1$, we do not use Taylor-series expansions in $\kappa$ for the coefficients, as defined by Eqs. (12)-(20). Instead we fit the potential energy functional forms, Eqs. (11) and (18), directly to the simulation data for each $\kappa$ value separately. For $\kappa=1.2$ and 1.4 , the $u / \Gamma$ values for the fluid phase, bcc solid phase, and fcc solid phase are given in Tables II, III, and IV, respectively. Note that $u / \Gamma \rightarrow-\kappa / 2$ as $\Gamma \rightarrow 0$ (in the fluid phase). The value $-\kappa / 2$ represents the energy of the Debye sheaths. ${ }^{19}$

Least-squares fitting of the functional forms to these data $(\Gamma \geqslant 1)$ yields the coefficient values shown in Tables $\mathrm{V}$ and VI. Since for $\kappa>1.066$ the fcc lattice becomes more stable than the bcc lattice at zero temperature, we have fitted the solid-phase function (18) to the data of bcc and fcc lattices separately. The numerical values of $f_{1}(\kappa)$, which are listed in Table VII, are obtained from a Simpson-rule numerical

TABLE VII. $f_{1}(\kappa)=f_{\text {fluid }}(\kappa, 1)-f_{\text {ideal }}(1)$ defined by Eq. (17).

\begin{tabular}{cc}
\hline$\kappa$ & $f_{1}(\kappa)$ \\
\hline 0.00 & -0.436765 \\
0.20 & -0.449484 \\
0.40 & -0.480913 \\
0.60 & -0.528365 \\
0.80 & -0.586650 \\
1.00 & -0.654089 \\
1.20 & -0.730380 \\
1.40 & -0.810280 \\
\hline \hline
\end{tabular}


TABLE VIII. Madelung energies for bcc and fcc Yukawa lattices. Madelung energies for $\kappa \leqslant 1.2$ are listed in Table III of Ref. 9.

\begin{tabular}{ccc}
\hline \hline$\kappa$ & $E_{\mathrm{bcc}}$ & $E_{\mathrm{fcc}}$ \\
\hline 1.2 & -1.03929199 & -1.03930236 \\
1.4 & -1.08834967 & -1.08837441 \\
\hline \hline
\end{tabular}

quadrature of $u / \Gamma(0 \leqslant \Gamma \leqslant 1)$ given in Table IV of Ref. 9 for $\kappa \leqslant 1.0$, and in Table II for $\kappa=1.2$ and 1.4. Table VIII gives the Madelung energies for bcc and fcc Yukawa lattices for $\kappa=1.2$ and 1.4 .

For a given $\kappa$, the intersection of the free energies of the fluid and solid phases gives the transition (i.e., melting or freezing) $\Gamma$ value (i.e., $\Gamma_{m}$ ). In Table IX we list $\Gamma_{m}$ for various $\kappa$ values. As mentioned above, we have used the dualvalue ( $\Gamma$ and $\kappa$ ) fitting of the free energies for $\kappa \leqslant 1.0$ and the single-value (i.e., $\Gamma$ only) fitting for $\kappa=1.2$ and 1.4 separately. To check the consistency of this approach, we also applied the single-value fitting method to the cases $\kappa \leqslant 1$, and found that it gave $\Gamma_{m}$ values very similar to those from the dual-value fit. For example, $\Gamma_{m}$ for $\kappa=1.0$ obtained from the single-value fit is 217.8 , whereas $\Gamma_{m}$ from the dual-value fit is 217.4. Similarly, for the OCP case $(\kappa=0)$, the single-value fit gives 171.2 while the dual-value fit gives 171.8. These values are essentially in very good agreement with most recent $\Gamma_{m}$ estimates for the OCP system. ${ }^{16,23,24}$

Figure 1 shows the phase diagram in the $\kappa-\Gamma$ plane. The closed circles represent the data points given in Table IX and the solid curve is the least-squares fit given by

$$
\Gamma_{m}=171.8+42.46 \kappa^{2}+3.841 \kappa^{4} \text { for } \kappa \leqslant 1.4 \text {. }
$$

\section{CONCLUDING REMARKS}

Earlier studies ${ }^{25-28}$ have employed normalizations different from Eq. (5) to represent the particulate temperature $T$ and the Debye screening length $\lambda_{D}$. For example, one may use $\rho=n^{-1 / 3}$, instead of the Wigner-Seitz radius $a$, as the length unit, and define $K=\rho / \lambda_{D}$. Kremer, Robbins, and Grest $^{25}$ normalized the temperature $T$ by the typical phonon energy of the fcc Yukawa lattice according to

$$
\mathscr{T}=\frac{k T}{m \omega_{E}^{2} \rho^{2}},
$$

TABLE IX. Transition values of $\Gamma$ and $\mathscr{T}$ at the fluid-solid phase boundary. These data are plotted in Figs. 1 and 2.

\begin{tabular}{ccc}
\hline \hline$\kappa$ & $\Gamma_{m}$ & $\mathscr{T}_{m}$ \\
\hline 0.0 & 171.8 & $2.240 \times 10^{-3}$ \\
0.2 & 173.5 & $2.267 \times 10^{-3}$ \\
0.4 & 178.6 & $2.332 \times 10^{-3}$ \\
0.6 & 187.1 & $2.425 \times 10^{-3}$ \\
0.8 & 199.6 & $2.535 \times 10^{-3}$ \\
1.0 & 217.4 & $2.647 \times 10^{-3}$ \\
1.2 & 243.3 & $2.736 \times 10^{-3}$ \\
1.4 & 268.8 & $2.907 \times 10^{-3}$ \\
\hline \hline
\end{tabular}

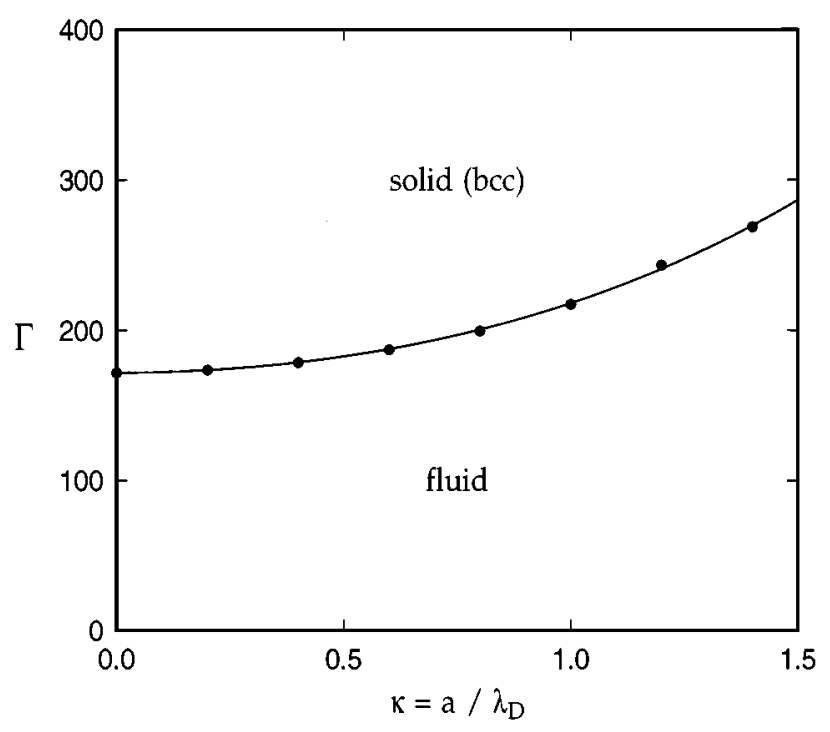

FIG. 1. Phase diagram of Yukawa systems as a function of the dimensionless inverse screening length $\kappa$ and the dimensionless inverse temperature $\Gamma$ defined in Eq. (3). The solid circles are fluid-solid phase boundary points obtained from MD simulations (see Table IX) and the solid curve is their least-squares fit. Note that the fcc solid phase is off-scale in this figure.

where $\omega_{E}$ is the Einstein frequency for the fcc Yukawa lattice, defined by

$$
\omega_{E}^{2}=\frac{2 k_{D}^{2}}{3 m} \sum_{i \neq j} \phi\left(\left|\mathbf{r}_{i}-\mathbf{r}_{j}\right|\right)
$$

with all particles at the fcc lattice sites. The Einstein frequency is related to the Madelung energy $E_{\mathrm{fcc}}(\kappa)$ in units of $Q^{2} / 4 \pi \epsilon_{0} a$ by

$$
\frac{m \omega_{E}^{2} a^{2}}{Q^{2} / 4 \pi \epsilon_{0} a}=\frac{2}{3} \kappa^{2}\left[E_{\mathrm{fcc}}(\kappa)+\frac{3}{2 \kappa^{2}}+\frac{\kappa}{2}\right] .
$$

It follows from Eqs. (3), (22), and (23) that the dimensionless temperature $\mathscr{T}$ is related to $\kappa$ and $\Gamma$ as

$$
\mathscr{T}=\frac{1}{\Gamma}\left(\frac{3}{4 \pi}\right)^{2 / 3}\left[\frac{2}{3} \kappa^{2} E_{\mathrm{fcc}}(\kappa)+\frac{\kappa^{3}}{2}+1\right]^{-1} .
$$

Equation (23) evidently becomes 1 , and $\mathscr{T}=(3 / 4 \pi)^{2 / 3} / \Gamma$, in the OCP limit $\kappa \rightarrow 0$. The Einstein phonon energies for the fcc Yukawa lattice in units of $Q^{2} / 4 \pi \epsilon_{0} \rho$, i.e.,

$$
\Omega^{2}=\frac{m \omega_{E}^{2} \rho^{2}}{Q^{2} / 4 \pi \epsilon_{0} \rho}
$$

are given in Table $\mathrm{X}$.

Table IX also shows $\mathscr{T}_{m}$, i.e., the dimensionless temperature $\mathscr{T}$ at the fluid-solid phase transition. These are plotted in Fig. 2 as functions of $\kappa$ or $K\left[=(4 \pi / 3)^{1 / 3} \kappa\right.$ $\approx 1.61199 \kappa]$. Here, the solid curve represents the leastsquares fit of the $\mathscr{T}_{m}$ values, given by

$$
\mathscr{T}_{m}=0.002240+0.000181 \kappa+0.000209 \kappa^{2}
$$

for $\kappa \leqslant 1.4$. 
TABLE X. Normalized squares of Einstein frequencies defined by Eq. (25).

\begin{tabular}{cc}
\hline \hline$\kappa$ & $\Omega^{2}$ \\
\hline 0.0 & 4.18879020 \\
0.2 & 4.09942706 \\
0.4 & 3.87048594 \\
0.6 & 3.55258586 \\
0.8 & 3.18604083 \\
1.0 & 2.80173848 \\
1.2 & 2.42225060 \\
1.4 & 2.06330814 \\
\hline \hline
\end{tabular}

The broken line is the fluid-solid phase boundary line estimated by Stevens and Robbins, ${ }^{28}$

$$
\mathscr{T}_{m}=0.0022+0.00022 \mathrm{~K} \text {, }
$$

which is an extrapolation of simulation data for $K>2.0$, matched linearly to the OCP phase-transition value given in Ref. 14. This linear extrapolation is in reasonable agreement with our results within the studied domain $0 \leqslant \kappa \leqslant 1.4$.

Interpolation formulas such as Eqs. (21) and (26) should not be used to extrapolate the function values as they can be grossly erroneous outside their domains of validity (i.e., $0 \leqslant \kappa \leqslant 1.4$ ). Indeed our preliminary simulation for larger $\kappa$ indicates that the quadratic dependence of $\mathscr{T}_{m}$ on $\kappa$ given in Eq. (21) does not continue for $\kappa>1.4$ : the dependence of $\mathscr{T}_{m}$ on $\kappa$ becomes linear for larger $\kappa$ values, similar to the result

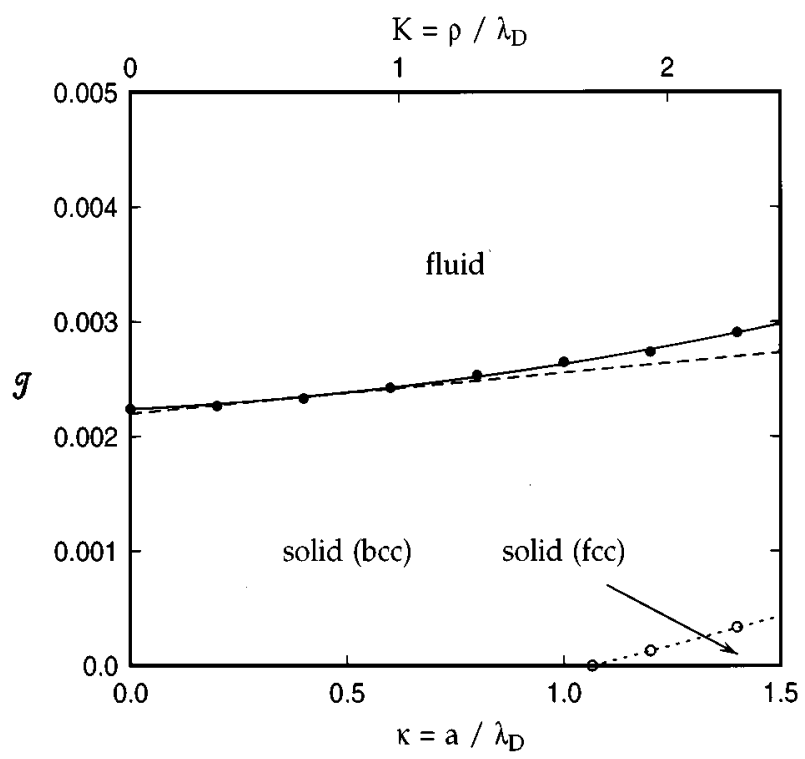

FIG. 2. Phase diagram of Yukawa systems as a function of the dimensionless inverse screening length $\kappa$ and the temperature $\mathscr{T}$ normalized by the Einstein phonon energy, defined by Eq. (22). $\mathscr{T}$ is related to $\Gamma$ through Eq. (24). The closed circles are fluid-solid phase boundary points obtained from MD simulations (see Table IX) and the solid line represents their quadratic least-squares fit. The broken line is the phase boundary suggested by Stevens and Robbins in Ref. 28. The dotted line is the bcc-fcc phase boundary obtained by Robbins, Kremer, and Grest (Ref. 26) based on the quasiharmonic theory. The open circles on the dotted line are bcc-fcc phase boundary points obtained from MD simulations (see Table XI).
TABLE XI. Transition values of $\Gamma$ and $\mathscr{T}$ at the bcc-fcc phase boundary. These values are plotted in Fig. 2, but are off-scale in Fig. 1.

\begin{tabular}{ccl}
\hline \hline$\kappa$ & $\Gamma_{s}$ & \multicolumn{1}{c}{$\mathscr{T}_{s}$} \\
\hline 1.066 & $\infty$ & 0.0 \\
1.2 & 5070 & $1.313 \times 10^{-4}$ \\
1.4 & 2325 & $3.361 \times 10^{-4}$ \\
\hline \hline
\end{tabular}

of Stevens and Robbins given by Eq. (27). For example, the Monte Carlo (MC) simulation results by Meijer and Frenkel $^{27}$ [which are consistent with Eq. (27)] show $\mathscr{T}_{m}=2.9 \times 10^{-3}$ for $\kappa=1.82(K=2.94)$ and $\mathscr{T}_{m}=3.1 \times 10^{-3}$ for $\kappa=2.39(K=3.85)$. Our preliminary MD simulation also shows $\mathscr{T}_{m} \simeq 3.1 \times 10^{-3}$ for $\kappa=1.82$ and $\mathscr{T}_{m} \simeq 3.2 \times 10^{-3}$ for $\kappa=2.39$, which are in good agreement with the earlier results. More details of our MD simulation for $\kappa>1.4$ will be presented in a future publication.

Table XI lists transition values of $\Gamma$ and $\mathscr{T}$ at the bccfcc phase boundary, i.e., the intersection of the bcc and fcc solid free energies. These transition temperatures are also plotted in Fig. 2. The dotted line represents the phase transition curve obtained from the quasiharmonic theory, ${ }^{26}$ which is in excellent agreement with our simulation results. Note that the bcc-fcc phase transition curve is off-scale in Fig. 1.

In summary, we have obtained the fluid-solid phase boundary curve of the Yukawa system in the weakly screened regime $(\kappa \leqslant 1.4)$. Unlike other MD or MC methods in earlier studies, where interparticle forces are calculated by pairwise summation over particles within a cut-off radius, our MD simulations use interparticle potentials summed over all particles, including all periodic images of particles residing in the cubical simulation box. Thus, long-range particle interactions are accurately taken into account in this method for the entire range of $\kappa$, including the unscreened (i.e., OCP) limit, $\kappa=0$. Our MD simulation method thus fills the gap between earlier extensive studies of the OCP system and strongly screened Yukawa systems.

Earlier estimates of the melting/freezing phase boundary for the Yukawa system in the weak-screening regime ${ }^{9}$ were based on the approximate value of the harmonic entropy constant, $\Sigma(\kappa)=-2.4938$ for all $\kappa \leqslant 1$ (which is exact only for $\kappa=0)$. In the present study, we have recalculated $\Gamma_{m}$ using correct $\Sigma(\kappa)$ values that are obtained from lattice dynamics calculations. The new $\Gamma_{m}$ values are presented in Table IX and Fig. 1, and are found to be systematically lower than the earlier approximate $\Gamma_{m}$ estimates in Ref. 9.

It is known that the entropy change per particle at the fluid-solid phase transition-i.e., $\Delta s=s_{\text {fluid }}-s_{\text {solid }}$, where $s=S / N k$ with $F=U-S T$-is almost constant over a wide temperature range. Recently Rosenfeld assumed $\Delta s=0.75$ (the OCP result from Refs. 14 and 29) and used the energy data from MD simulations by Farouki and Hamaguchi ${ }^{9}$ to obtain the melting $\Gamma$ values in the weak screening region $(\kappa \leqslant 1),{ }^{30}$ which are in good agreement with our $\Gamma_{m}$ values shown in Table IX and Fig. 1. We directly calculate the entropy change $\Delta s$ from our MD simulations, using the relation $\Delta u-\Delta s=f_{\text {fluid }}-f_{\text {solid }}=0$ at $\Gamma=\Gamma_{m}$. Table XII shows 
TABLE XII. Entropy jump $\Delta s$ at the fluid-solid phase transition.

\begin{tabular}{cc}
\hline \hline$\kappa$ & $\Delta s$ \\
\hline 0.0 & 0.745 \\
0.2 & 0.745 \\
0.4 & 0.745 \\
0.6 & 0.744 \\
0.8 & 0.741 \\
1.0 & 0.737 \\
1.2 & 0.750 \\
1.4 & 0.717 \\
\hline \hline
\end{tabular}

$\Delta s$ as a function of $\kappa$, which indicates $\Delta s \simeq 0.75$ and corroborates Rosenfeld's assumption.

We have also estimated the bcc-fcc phase boundary at $\kappa=1.2$ and 1.4 (Table XI), using the same MD simulation method. The obtained transition temperatures $\mathscr{T}_{s}$ show excellent agreement with the results of quasiharmonic theory ${ }^{26}$ in this regime.

\section{ACKNOWLEDGMENTS}

The authors acknowledge useful discussions with H. E. DeWitt and Y. Rosenfeld, who helped resolve the discrepancy in the $\Gamma_{m}$ values given in Ref. 9.

${ }^{1}$ H. Ikezi, Phys. Fluids 29, 1764 (1986).

${ }^{2}$ R. T. Farouki and S. Hamaguchi, Appl. Phys. Lett. 61, 2973 (1992).

${ }^{3}$ Y. Hayashi and K. Tachibana, Jpn. J. Appl. Phys. 33, L804 (1994).

${ }^{4}$ H. Thomas, G. E. Morfill, V. Demmel, J. Goree, B. Feuerbacher, and D. Möhlmann, Phys. Rev. Lett. 73, 652 (1994).

${ }^{5}$ J. H. Chu and I. Lin, Physica A 205, 183 (1994).
${ }^{6}$ J. H. Chu and I. Lin, Phys. Rev. Lett. 72, 4009 (1994).

${ }^{7}$ Th. Trottenberg, A. Melzer, and A. Piel, Plasma Sources Sci. Technol. 4, 450 (1995).

${ }^{8}$ C. A. Murray and R. A. Wenk, in Strongly Coupled Plasma Physics, edited by H. M. Van Horn and S. Ichimaru (University of Rochester Press, Rochester, 1993).

${ }^{9}$ R. T. Farouki and S. Hamaguchi, J. Chem. Phys. 101, 9885 (1994).

${ }^{10}$ S. G. Brush, H. L. Sahlin, and E. Teller, J. Chem. Phys. 45, 2102 (1966).

${ }^{11}$ J.-P. Hansen, Phys. Rev. A 8, 3096 (1973).

${ }^{12}$ H. E. DeWitt, Phys. Rev. A 14, 1290 (1976).

${ }^{13}$ M. Baus and J.-P. Hansen, Phys. Rep. 59, 1 (1980).

${ }^{14}$ G. S. Stringfellow, H. E. DeWitt, and W. L. Slattery, Phys. Rev. A 41, 1105 (1990).

${ }^{15}$ Strongly Coupled Plasma Physics, edited by F. J. Rogers and H. E. DeWitt (Plenum, New York, 1986).

${ }^{16}$ R. T. Farouki and S. Hamaguchi, Phys. Rev. E 47, 4330 (1993).

${ }^{17}$ R. T. Farouki and S. Hamaguchi, J. Comput. Phys. 115, 276 (1994).

${ }^{18}$ S. Hamaguchi and R. T. Farouki, J. Chem. Phys. 101, 9876 (1994).

${ }^{19}$ S. Hamaguchi and R. T. Farouki, Phys. Rev. E 49, 4430 (1994).

${ }^{20}$ A. A. Maradudin, E. W. Montroll, G. H. Weiss, and I. P. Ipatova, Theory of Lattice Dynamics in the Harmonic Approximation, 2nd ed. (Academic, New York, 1971).

${ }^{21}$ D. H. E. Dubin and H. E. DeWitt, Phys. Rev. B 49, 3043 (1994).

${ }^{22}$ Note that there are some typographical errors in the fitting parameters given in Ref. 9. The signs of $\delta a_{2}, b_{0}, b_{1}, d_{1}$ in Eqs. (17) and (18) of Ref. 9 should read plus, instead of minus.

${ }^{23}$ D. H. E. Dubin, Phys. Rev. A 42, 4972 (1990).

${ }^{24}$ H. Nagara, Y. Nagata, and T. Nakamura, Phys. Rev. A 36, 1859 (1987).

${ }^{25}$ K. Kremer, M. O. Robbins, and G. S. Grest, Phys. Rev. Lett. 57, 2694 (1986).

${ }^{26}$ M. O. Robbins, K. Kremer, and G. S. Grest, J. Chem. Phys. 88, 3286 (1988).

${ }^{27}$ E. J. Meijer and D. Frenkel, J. Chem. Phys. 94, 2269 (1991).

${ }^{28}$ M. J. Stevens and M. O. Robbins, J. Chem. Phys. 98, 2319 (1993).

${ }^{29}$ W. L. Slattery, G. Doolen, and H. E. DeWitt, Phys. Rev. A 21, 2087 (1980).

${ }^{30}$ Y. Rosenfeld, J. Chem. Phys. 103, 9800 (1995). 